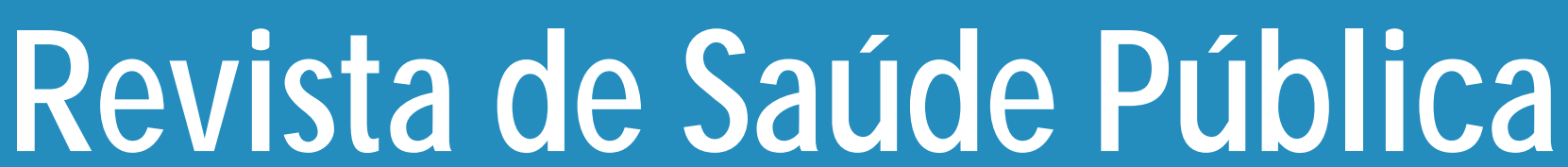
Journal of Public Health

\title{
Preferências por alimentos doces e cárie dentária em pré-escolares
}

\section{Taste preference for sweetness and caries prevalence in preschoolchildren}

\section{Nilce E Tomita, Paulo Nadanovsky, Ana Luiza F Vieira** e Eymar S Lopes}

Departamento de O dontopediatria, O rtodontia e Saúde Coletiva da Faculdade de O dontologia de Bauru da Universidade de São Paulo. Bauru, SP - Brasil (NET, ESL), Instituto de M edicina Social da Universidade do Estado do Rio de Janeiro. Rio de Janeiro, RJ - Brasil (PN) 


\title{
Preferências por alimentos doces e cárie dentária em pré-escolares* Taste preference for sweetness and caries prevalence in preschoolchildren
}

\author{
Nilce E Tomita, Paulo Nadanovsky, Ana Luiza F Vieira** e Eymar S Lopes \\ Departamento de Odontopediatria, Ortodontia e Saúde Coletiva da Faculdade de Odontologia de \\ Bauru da Universidade de São Paulo. Bauru, SP - Brasil (NET, ESL), Instituto de Medicina Social da \\ Universidade do Estado do Rio de Janeiro. Rio de Janeiro, RJ - Brasil (PN)
}

\section{Descritores}

Cárie dentária, epidemiologia. Preferências alimentares. Sacarose na dieta, efeitos adversos.

\section{Keywords}

Dental caries, epidemiology. Food preferences. Dietary sucrose, adverse effects.

\section{Resumo}

\section{Objetivo}

Avaliar as preferências pelo sabor doce e sua associação com as condições socioeconômicas e a prevalência de cárie dentária em pré-escolares.

\section{Métodos}

A amostra foi composta por 572 crianças de 4 a 6 anos, matriculadas em pré-escolas, dividida em estratos socioeconômicos segundo a procedência (um bairro central, um periférico e Programa de Desfavelamento).

Estudo transversal, foi desenvolvido em duas etapas. A preferência pelo sabor doce foi avaliada através do Sweet Preference Inventory modificado. A concentração das soluções variou de 0 a 400 g/litro ( 0 a 1,17 molar). Foi utilizado o índice ceos para verificar a prevalência de cárie.

\section{Resultados}

Foram encontradas diferenças estatisticamente significantes entre as crianças do Programa de Desfavelamento e dos demais grupos na preferência pelo sabor doce e no consumo de açúcar, bem como associação entre a procedência e o percentual de crianças livres de cárie, sendo o grupo do Desfavelamento o menos favorecido $(\mathrm{p}<0,05)$. Conclusão

Os achados sustentam a hipótese de que o nível socioeconômico influencia a preferência por açúcar e esta, por sua vez, está associada à prevalência de cárie dentária na dentição decídua.

\begin{abstract}
Objective

To assess the preference for sweetness among preschool children and differences between less and more deprived groups. In addition, to assess whether sweet taste preference was associated with presence of caries.

Methods

The sample was composed by 572 preschool children aged between 4 and 6, distributed in three day nurseries of varied socioeconomic background.

Cross-sectional study developed in two steps. Preference for sweetness was assessed using a modified version of the Sweet Preference Inventory. The solutions varied in sugar concentration from 0 to 1,17 molar (0 to $400 \mathrm{~g} /$ litre). The presence of caries
\end{abstract}

Correspondência para/Correspondence to: Nilce E Tomita-Al. Octávio Pinheiro Brisolla, 9-75 - 17043-101 Bauru, SP - Brasil. E-mail: netomita@usp.br

\footnotetext{
*Trabalho desenvolvido no Departamento de Odontopediatria, Ortodontia e Saúde Coletiva da Faculdade de Odontologia de Bauru,USP Apresentado na 76th General Session of the International Association for Dental Research, em Nice-França, 1998.

**Aluna de pós-graduação em Odontopediatria da Faculdade de Odontologia de Bauru/USP

Edição subvencionada pela Fapesp (Processo n. 98/13915-5).

Recebido em 29.5.1998. Reapresentado em 4.5.1999. Aprovado em 24.6.1999.
} 
was assessed using the defs index. The socioeconomic status of the sample was classified according to the origin of domicile.

Results

The variation in preference for sweetness in our sample was too small. Most children preferred the sweetest juice. This reduced the ability of this variable to explain variation in caries prevalence. Despite this limitation, our results showed that socioeconomic level influenced preference for sweetness, which in turn was associated with caries prevalence.

Conclusion

The socioeconomic status influence the sweetness preference and this, in turn, is associated with the dental caries prevalence.

\section{INTRODUÇÃO}

Nas últimas décadas, o consumo de açúcar em sociedades emergentes tem sido crescente. A substituição de produtos locais por alimentos manufaturados, particularmente com alto conteúdo de açúcar, tem sido acompanhada por um aumento na cárie den$\operatorname{tal}^{13}$.

O consumo de alimentos adoçados é influenciado por uma variedade de fatores biológicos, psicológicos, sociais e ambientais. Uma preferência por doces tem sido sugerida como importante fator no consumo individual de açúcar ${ }^{3,12}$. Em 1910, Peterson, Rainey ${ }^{11}$ observaram que neonatais demonstravam uma reação facial de prazer a alimentos doces. Esta preferência também ocorreu com crianças maiores ${ }^{1}$.

A freqüência do consumo de açúcar é um importante fator na etiologia da cárie ${ }^{15}$. Entretanto, em estudos epidemiológicos, uma fraca correlação entre o consumo de açúcar e a prevalência de cárie tem sido encontrada ${ }^{2}$.

É possível que a demanda individual por açúcar seja limitada, mas que o ponto final esteja sujeito a grande variação individual ${ }^{4}$. Estudos que avaliaram os padrões de ingestão produziram resultados comparáveis com uma demanda "pico" por sacarose de concentração de 0,3 M (molar), com posterior declínio .

Outras variáveis para a preferência por açúcar incluem sexo, etnia e sensibilidade gustativa ${ }^{3}$.

Além dos estudos populacionais que têm relatado a associação entre os padrões de ingestão de açúcar e a prevalência de cárie, alguns estudos reportam a questão do paladar ou preferência por açúcares como uma variável de maior valor explicativo para a ocorrência de cárie em diferentes grupos.

Desor et al. ${ }^{3}$ demostraram, em 1975, que o paladar está relacionado com a sacarose na dieta. Entretanto, um indivíduo que tenha uma leve preferência por açúcar pode ter uma elevada ingestão diária de açúcar.
Catalanotto ${ }^{1}$, em1979, analisou a relação entre o paladar por sacarose e a suscetibilidade à cárie em crianças. Não houve diferença entre o grupo sem cárie e o grupo suscetível à cárie quanto à detecção de açúcar nas soluções.

Em 1983, Nilsson e Holm ${ }^{10}$ testaram o limiar do paladar e a preferência por açúcar. Houve uma diferença muito pequena entre as soluções com sacarose preferidas para os grupos com alta e com baixa quantidade de dentes restaurados.

Honkala et al. ${ }^{5}$, em 1984, analisaram os possíveis determinantes do consumo de açúcar: fatores sociodemográficos, hábitos dentais, desempenho escolar, dinheiro disponível na carteira e preferência por doce. O consumo de açúcar decresce com a idade. A alta correlação entre a preferência por doce das crianças e seus pais vem sendo observada, o que sugere que a preferência por doces é, em parte, um hábito aprendido.

Wync et al. ${ }^{15}$, em 1995, estudaram algumas características (nível socioeconômico, dieta, uso de chupeta adocicada) de crianças que desenvolveram cárie. A maior prevalência de cárie em crianças com nível social mais baixo concordou com estudos que atribuem esta condição à falta de orientação sobre o assunto, uma vez que os pais, precocemente, davam aos filhos alimentos doces e em alta freqüência ao dia.

Em estudos em que as pessoas são entrevistadas acerca de seus hábitos alimentares, a confiabilidade das respostas é questionável. As preferências pelo gosto doce supostamente retratam com maior fidelidade a frequiência do consumo diário de açúcar que o relato das próprias pessoas.

A partir desta hipótese, o presente estudo tem por objetivo avaliar os padrões de preferência gustativa pelo sabor doce por pré-escolares provenientes de variados estratos socioeconômicos e a associação entre essa preferência e a prevalência de cárie. 


\section{MÉTODOS}

A seleção da amostra foi feita através de sorteio de duas instituições públicas de ensino pré-escolar do município de Bauru, São Paulo. A primeira foi selecionada entre aquelas localizadas na região central do município e a segunda entre as instituições da periferia. $\mathrm{O}$ terceiro grupo foi composto por crianças de nível socieconômico mais baixo provenientes do Programa de Desfavelamento de Bauru. A totalidade de crianças de 4 a 6 anos presentes nas instituições e no Programa de Desfavelamento foi examinada no dia da visita. Constituíram a amostra do estudo 572 crianças de 4 a 6 anos de idade, de ambos os sexos, conforme Tabela 1.

A preferência por açúcar foi avaliada através de um indicador previamente desenvolvido e testado em populações adultas, o Sweet Preference Inventory ${ }^{6}$. Este indicador avalia a preferência por 5 diferentes concentrações de uma solução açucarada: $0,0 \mathrm{M}$ (sem açúcar); 0,15 M; 0,29 M;0,44 M; 0,59 M.

Após a utilização deste indicador no estudo piloto, algumas modificações foram efetuadas. O chá do teste original foi substituído por suco de uva, considerando o clima do Brasil e os hábitos preferenciais de crianças em idade pré-escolar por suco de frutas.

A ampla preferência das 30 crianças examinadas no estudo-piloto $(74,4 \%)$ pela solução com maior concentração de açúcar sugeriu que o aumento das concentrações das 4 soluções adoçadas poderia oferecer maior possibilidade de discriminação para as análises propostas (Tabela 2).

Embora nenhuma das crianças tenha gostado do suco sem açúcar no estudo-piloto, foi decidido mantê-lo no teste para servir como uma referência.

O suco de uva com as diferentes concentrações de açúcar era preparado, no momento do exame, com auxílio de um liquidificador. A seguir, era acondicionado em cinco galões térmicos, identificados por letras.

Para a realização do teste, cada criança recebia o suco em copinhos plásticos descartáveis (contendo $10 \mathrm{ml}$ ), em sequiência crescente de concentração de açúcar. Antes de todas as degustações, para "neutralizar" o paladar, as crianças ingeriam um pedaço de bolacha de água e sal. Entre uma degustação e outra era questionado qual
Tabela 2 - Soluções de suco de uva* e respectivas concentrações de açúcar refinado. Bauru-SP, 1997.

\begin{tabular}{cc}
\hline Solução & $\begin{array}{c}\text { Concentração de açúcar } \\
\text { (Molar }=\text { g/litro) }\end{array}$ \\
\hline A & $0 M$ \\
B & $0,29 M=100 \mathrm{~g} /$ litro \\
C & $0,59 M=200 \mathrm{~g} /$ litro \\
D & $0,77 M=300 \mathrm{~g} /$ litro \\
E & $1,17 \mathrm{M}=400 \mathrm{~g} /$ litro \\
\hline$*$ Suco de uva, preparado segundo recomendações do
\end{tabular}
fabricante.

solução a criança preferia e ao final do teste ela apontava a solução de sua escolha.

Utilizou-se o índice ceos $^{8}$ para determinar a prevalência de cárie na dentição decídua. O exame foi realizado antes do teste do açúcar, para que a bolacha não atrapalhasse a visualização das superfícies oclusais e para evitar tendenciosidade do examinador. Este exame foi realizado sob luz natural, com espelho plano e sonda exploradora, quando necessário.

$\mathrm{Na}$ análise dos resultados, as diferenças entre os grupos foram avaliadas por testes bivariados de associação (teste do qui-quadrado), em um nível de significância estatística de $5 \%(\mathrm{p}<0,05)$.

\section{RESULTADOS}

O comportamento quanto à escolha da solução foi bastante semelhante entre meninos e meninas. Nos resultados agrupados (Tabela 3), observa-se a distribuição da amostra segundo a preferência por açúcar e a procedência. Houve predominância da solução mais concentrada em açúcar, em todas as idades e em cada grupo. À análise bivariada, ao agrupar-se as soluções menos concentradas (A, B, C e D) e comparar sua distribuição com a solução mais adocicada (E), observa-se que o grupo Desfavelamento é o discrepante $(\mathrm{p}<0,005)$; a preferência pela solução mais doce foi significantemente maior que nos outros grupos.

A prevalência de cárie apresentou tendência crescente com a idade, em todos os grupos. As crianças do Desfavelamento apresentaram os valores ceos mais elevados (Tabela 4)

O número e percentual de crianças isentas de cárie, em todas as idades e para crianças de ambos os sexos, foi significantemente menor no grupo Desfavelamento que nos demais grupos (Tabela 5).

Tabela 1 - Distribuição da amostra segundo a idade e sexo, por procedência. Bauru-SP, 1997.

\begin{tabular}{|c|c|c|c|c|c|c|c|c|c|c|c|c|c|c|c|c|c|c|}
\hline \multirow[b]{4}{*}{ Procedência } & \multirow{2}{*}{\multicolumn{6}{|c|}{$\frac{\text { Masculino }}{\text { Idade }}$}} & \multirow{2}{*}{\multicolumn{6}{|c|}{$\frac{\text { Feminino }}{\text { Idade }}$}} & \multirow{2}{*}{\multicolumn{6}{|c|}{$\frac{\text { Total }}{\text { Idade }}$}} \\
\hline & & & & & & & & & & & & & & & & & & \\
\hline & \multicolumn{2}{|l|}{4} & \multicolumn{2}{|c|}{5} & \multirow{2}{*}{$\begin{array}{c}6 \\
N\end{array}$} & \multirow[b]{2}{*}{$\%$} & \multirow{2}{*}{$\begin{array}{l}4 \\
\mathrm{~N}\end{array}$} & \multirow{2}{*}{\multicolumn{2}{|c|}{$\begin{array}{l}5 \\
N\end{array}$}} & \multirow[b]{2}{*}{$\%$} & \multirow{2}{*}{$\begin{array}{l}6 \\
N \\
\end{array}$} & \multirow[b]{2}{*}{$\%$} & \multirow{2}{*}{$\begin{array}{l}4 \\
N\end{array}$} & \multirow[b]{2}{*}{$\%$} & \multirow{2}{*}{$\begin{array}{r}5 \\
\mathrm{~N} \\
\end{array}$} & \multirow[b]{2}{*}{$\%$} & \multirow{2}{*}{$\begin{array}{l}6 \\
N \\
\end{array}$} & \multirow[b]{2}{*}{$\%$} \\
\hline & $\mathrm{N}$ & $\%$ & $\mathrm{~N}$ & $\%$ & & & & & & & & & & & & & & \\
\hline Região central & 32 & 38,1 & 46 & 59,0 & 39 & 31,0 & 23 & 31,5 & 51 & 50,5 & 41 & 37,3 & 55 & 35,0 & 97 & 54,2 & 80 & 33,9 \\
\hline Periferia & 19 & 22,6 & 18 & 23,1 & 71 & 56,3 & 19 & 26,0 & 31 & 30,7 & 56 & 50,9 & 38 & 24,2 & 49 & 27,4 & 127 & 53,8 \\
\hline Desfavelamento & 33 & 39,3 & 14 & 17,9 & 16 & 12,7 & 31 & 42,5 & 19 & 18,8 & 13 & 11,18 & 64 & 40,8 & 33 & 18,4 & 29 & 12,3 \\
\hline Total & 84 & 100,0 & 78 & 100,0 & 126 & 100,0 & 73 & 100,0 & 101 & 100,0 & 110 & 100,0 & 157 & 100,0 & 179 & 100,0 & 236 & 100,0 \\
\hline
\end{tabular}


Tabela 3 - Distribuição da amostra segundo a preferência por açúcar e idade, por procedência. Bauru-SP, 1997.

\begin{tabular}{|c|c|c|c|c|c|c|c|c|c|c|c|c|c|c|c|c|c|c|}
\hline \multirow[b]{4}{*}{ Solução } & \multirow{2}{*}{\multicolumn{6}{|c|}{$\frac{\text { Região Central }}{\text { Idade }}$}} & \multirow{2}{*}{\multicolumn{6}{|c|}{$\begin{array}{c}\text { Periferia } \\
\text { Idade }\end{array}$}} & \multirow{2}{*}{\multicolumn{6}{|c|}{$\frac{\text { Desfavelamento }}{\text { Idade }}$}} \\
\hline & & & & & & & & & & & & & & & & & & \\
\hline & 4 & \multicolumn{3}{|c|}{5} & \multirow{2}{*}{$\begin{array}{c}6 \\
N\end{array}$} & \multirow[b]{2}{*}{$\%$} & \multirow{2}{*}{$\begin{array}{l}4 \\
\mathrm{~N}\end{array}$} & \multirow[b]{2}{*}{$\%$} & \multirow{2}{*}{$\begin{array}{l}5 \\
N\end{array}$} & \multirow[b]{2}{*}{$\%$} & \multirow{2}{*}{$\begin{array}{l}6 \\
N\end{array}$} & \multirow[b]{2}{*}{$\%$} & \multirow{2}{*}{$\begin{array}{l}4 \\
\mathrm{~N}\end{array}$} & \multirow[b]{2}{*}{$\%$} & \multirow{2}{*}{$\begin{array}{l}5 \\
N\end{array}$} & Idade & \multirow{2}{*}{$\begin{array}{r}6 \\
N \\
\end{array}$} & \multirow[b]{2}{*}{$\%$} \\
\hline & $\mathrm{N}$ & $\%$ & $\mathrm{~N}$ & $\%$ & & & & & & & & & & & & $\%$ & & \\
\hline A & 1 & 1,8 & 10 & 10,3 & 5 & 6,3 & 1 & 2,6 & 2 & 4,1 & 9 & 7,1 & 0 & - & 0 & - & 0 & - \\
\hline B & 1 & 1,8 & 1 & 1,0 & 5 & 6,3 & 4 & 10,5 & 1 & 2,0 & 3 & 2,4 & 2 & 3,1 & 1 & 3,0 & 0 & - \\
\hline C & 3 & 5,5 & 7 & 7,2 & 2 & 2,4 & 1 & 2,6 & 1 & 2,0 & 4 & 3,1 & 1 & 1,6 & 1 & 3,0 & 3 & 10,3 \\
\hline $\mathrm{D}$ & 7 & 12,7 & 11 & 11,4 & 8 & 10,0 & 4 & 10,5 & 3 & 6,1 & 7 & 5,5 & 3 & 4,7 & 1 & 3,0 & 2 & 6,9 \\
\hline E & 43 & 78,2 & 68 & 70,1 & 60 & 75,0 & 28 & 73,8 & 42 & 85,8 & 104 & 81,9 & 58 & 90,6 & 30 & 91,0 & 24 & 62,8 \\
\hline Total & 55 & 100,0 & 97 & 100,0 & 80 & 100,0 & 38 & 100,0 & 49 & 100,0 & 127 & 100,0 & 64 & 100,0 & 33 & 100,0 & 29 & 100,0 \\
\hline
\end{tabular}

Níveis de concentração de açúcar: $\underline{\mathrm{A}}(0,0 \mathrm{~m}$ - sem açúcar); $\underline{B}=0,15 \mathrm{~m} ; \underline{\mathrm{C}}=0,29 \mathrm{~m} ; \underline{\mathrm{D}}=0,44 \mathrm{~m}$ e $\underline{\mathrm{E}}=0,59 \mathrm{~m}$ $x^{2}=12,14^{*} \quad p=0,00232$

Entretanto, ao testar a associação entre o número de crianças isentas de cárie e a preferência por açúcar, não foram observadas diferenças estatisticamente significantes (Tabela 6). Para a análise, foram agrupadas as 4 soluções de menores concentrações de açúcar para comparação com a solução mais adocicada e sua distribuição entre as crianças que não apresentavam cárie.

\section{DISCUSSÃO}

A preferência por sabores doces pode ser detectada nos recém-nascidos ${ }^{11}$. A discriminação quanto à preferência, porém, ocorre com o tempo e é afetada por inúmeros fatores.

No presente estudo, verificou-se que crianças a partir dos 4 anos de idade apresentam a capacidade

Tabela 4 - Índice ceos para as crianças da amostra segundo a idade e procedência. Bauru-SP, 1997.

\begin{tabular}{cccccccccc} 
Idade & \multicolumn{3}{c}{ Região central } & \multicolumn{3}{c}{ Periferia } & \multicolumn{4}{c}{ Programa de } \\
& & & & & \multicolumn{4}{c}{ Desfavelamento } \\
(anos) & ceos & DP & N & ceos & DP & N & ceos & DP & $N$ \\
\hline 4 & 3,09 & 6,79 & 55 & 1,71 & 3,58 & 38 & 4,20 & 7,29 & 64 \\
5 & 3,06 & 4,97 & 97 & 2,73 & 5,67 & 49 & 6,33 & 8,32 & 33 \\
6 & 5,01 & 7,24 & 80 & 4,56 & 5,86 & 126 & 9,90 & 11,60 & 29 \\
\hline
\end{tabular}

DP = Desvio-padrão

N: Número de crianças discriminatória para apontar suas preferências gustativas por açúcar. A maioria das crianças apontou sua preferência pela solução mais doce (Tabela 3 ), sem variação segundo a idade e o sexo.

A discriminação necessária para justificar o uso do Sweet Preference Inventory modificado foi adequada no estudo piloto. Uma proporção muito alta de crianças nas escolas públicas e do grupo de Desfavelamento escolheu o suco com a mais alta concentração de açúcar como sua primeira opção.

$\mathrm{Na}$ literatura, relata-se que os indivíduos do sexo masculino têm maior preferência por açúcar que os do sexo feminino $0^{4,5,10}$. No presente estudo, verifica-se que, em idade pré-escolar, esta diferença não é evidente.

Os efeitos dos fatores socioeconômicos no consumo de açúcar têm sido relatados por alguns estudos ${ }^{2}$. Foi observada uma associação negativa entre o estrato socioeconômico e a preferência pelo suco mais adocicado. Embora a solução mais concentrada tenha recebido a preferência da maioria das crianças avaliadas, no grupo Desfavelamento esta preferência destaca-se dos demais grupos (Tabela 3). A consciência dos efeitos prejudiciais do açúcar afetam o padrão de con-

Tabela 5 - Número e percentual de crianças isentas de cárie segundo o sexo e idade, por procedência. Bauru-SP, 1997.

\begin{tabular}{|c|c|c|c|c|c|c|c|c|c|c|c|c|}
\hline \multirow[b]{2}{*}{ Procedência } & \multicolumn{6}{|c|}{$\begin{array}{c}\text { Masculino } \\
\text { Idade }\end{array}$} & \multicolumn{6}{|c|}{$\begin{array}{l}\text { Feminino } \\
\text { Idade }\end{array}$} \\
\hline & $N^{4}$ & $\%$ & $\mathrm{~N}^{5}$ & $\%$ & $\mathrm{~N}^{6}$ & $\%$ & $N^{4}$ & $\%$ & $\mathrm{~N}^{5}$ & $\%$ & ${ }^{6}$ & $\%$ \\
\hline Região Central & 18 & 40,0 & 5 & 38,5 & 21 & 36,8 & 15 & 36,6 & 26 & 48,1 & 19 & 44,1 \\
\hline Periferia & 14 & 31,1 & 6 & 46,1 & 33 & 57,9 & 13 & 31,7 & 21 & 38,9 & 21 & 48,9 \\
\hline Desfavelamento & 13 & 28,9 & 2 & 14,4 & 3 & 5,3 & 13 & 31,7 & 7 & 13,0 & 3 & 7,0 \\
\hline Total & 45 & 100,0 & 13 & 100,0 & 57 & 100,0 & 41 & 100,0 & 54 & 100,0 & 43 & 100,0 \\
\hline
\end{tabular}

Tabela 6 - Número e percentual de crianças livres de cárie segundo a preferência por açúcar, por sexo e idade. Bauru-SP, 1997.

\begin{tabular}{|c|c|c|c|c|c|c|c|c|c|c|c|c|}
\hline \multirow{4}{*}{ Solução } & \multirow{2}{*}{\multicolumn{7}{|c|}{ Masculino }} & \multicolumn{5}{|c|}{ Feminino } \\
\hline & & & & Idade & & & & & & ade & & \\
\hline & 4 & & 5 & & 6 & & 4 & & 5 & & 6 & \\
\hline & $\mathrm{N}$ & $\%$ & $\mathrm{~N}$ & $\%$ & $\mathrm{~N}$ & $\%$ & $\mathrm{~N}$ & $\%$ & $\mathrm{~N}$ & $\%$ & $\mathrm{~N}$ & $\%$ \\
\hline $\mathrm{A}$ & 0 & - & 3 & 60,0 & 4 & 100,0 & 2 & 100,0 & 3 & 42,9 & 2 & 33,3 \\
\hline B & 2 & 40,0 & 1 & 100,0 & 3 & 60,0 & 2 & 100,0 & 1 & 50,0 & 2 & 66,7 \\
\hline C & 4 & 100,0 & 2 & 66,7 & 3 & 42,9 & 1 & 100,0 & 3 & 50,0 & 1 & 50,0 \\
\hline D & 3 & 50,0 & 3 & 33,3 & 2 & 25,0 & 4 & 50,0 & 4 & 66,6 & 1 & 11,1 \\
\hline $\mathrm{E}$ & 36 & 52,2 & 24 & 40,0 & $4 \overline{5}$ & 45,9 & 32 & 53,3 & 43 & 53,8 & 37 & 41,1 \\
\hline Total & 45 & 53,6 & 33 & 42,3 & 57 & 45,2 & 41 & 56,2 & 54 & 53,5 & 43 & 39,1 \\
\hline
\end{tabular}


sumo, particularmente nos grupos com mais altos níveis de educação paterna ${ }^{5}$.

A história de cárie na dentição decídua das crianças examinadas apresenta menores valores ceos que dados verificados para crianças do município de Bauru, em inquérito realizado em $1991{ }^{14}$. Contudo, ao considerar o grupo Desfavelamento isoladamente (Tabela 4), observam-se valores ceos superiores aos relatados por Tomita et al. ${ }^{14}$. Estas diferenças podem ser devidas à situação de deprivação econômica apresentada por este grupo.

O percentual de crianças livres de cárie é também significantemente menor entre as crianças do grupo de Desfavelamento (Tabela 5). Algumas transformações ocorridas nos padrões alimentares de famílias oriundas de favelas, bem como a inexistência de condições de fluoretação das águas nos períodos anteriores podem estar associadas a este quadro.

Um padrão gustativo de preferência para soluções mais adoçadas e conseqüente maior consumo de açúcar tem sido relacionado a níveis mais altos de cárie ${ }^{6}$. Porém, a associação entre a ausência de cárie e a preferência por açúcar não foi observada no presente estudo (Tabela 6). Os percentuais de crianças livres de cárie que relataram preferir a solução mais doce oscilaram entre $40,0 \%$ e $53,3 \%$. Nos demais grupos, houve uma variação entre $11,1 \%$ e 100,0\% de isentas de cárie.

\section{REFERÊNCIAS}

1. Catalanotto FA, Gaulin-Kremer E, Shaw JL. Sucrose taste functions and dental caries in children. J Dent Res 1979;1327-32.

2. Clancy KI, Bibbi BG, Goldberg HI. Snack food intake of adolescents and caries development. J Dent Research 1977;45:568-73.

3. Desor JA, Greene LS, Maller O. Preference for sweet and salty in 9- to 15- year- olds and adult humans. Science 1975;190:686-7.

4. Ekman G, Akesson C. Saltness, sweetness and preference. A study of quantitative relations in individuals subjects. Scand J Psychol 1965;6:241-53.

5. Honkala E, Myyssönen V, Rempelä A. Determinants of frequency of children's sweets consumption. Acta Odontol Pediatr 1984;5:13-9.

6. Jamel HA, Sheiham A, Cowell CR, Watt RG. Taste preference for sweetness in urban and rural population in Iraq. J Dent Res 1996;75:1879-84.

7. Jordan H, Wieland W, Zebley S, Stellar E, Stunkard J. Direct measurement of food intake in man: a method for the objective study of eating behavior. Psychossomat Med 1966;28:836-42.
Esta distribuição heterogênea de crianças entre os mesmos pode ter trazido prejuízo à análise.

Embora uma preferência inata por doces seja relatada em diferentes estudos, não constitui uma entidade estática. Pelo contrário, a preferência por açúcar é altamente influenciada pela exposição aumentada e maior disponibilidade de açúcar associada à urbanização ${ }^{6}$. Isto tem importantes implicações para o desenvolvimento de políticas públicas com o objetivo de promover uma alimentação mais saudável ${ }^{9}$ e de orientações aos pais no que se refere à alimentação infantil.

Em conclusão, não houve associação entre a ausência de cárie e a preferência por açúcar. Por sua vez, as crianças do estrato socioeconômico menos favorecido (Desfavelamento) apresentaram maior preferência pelo açúcar e também maior prevalência de cárie, o que implica a necessidade de planejar ações direcionadas à educação em saúde e promoção de saúde bucal em populações com privações sociais.

\section{AGRADECIMENTOS}

Ao Departamento de Odontologia Social da Faculdade de Odontologia de Bauru, pelo apoio logístico, e à técnica em higiene dentária Marta Regina Liporacci Aoki, desse departamento, e às escolas públicas visitadaspela colaboração.

8. Klein H, Palmer CE, Knutson JW. Studies in on denta caries. I. Dental status and dental needs of elementary schoolchildren. Public Health Report 1938;53:751-65.

9. Love R, Kalnins I. Individualist and structuralist perspectives on nutrition education for Canadian children. Soc Sci Med 1984;18:199-204.

10. Nilsson B, Holm AR. Taste thresholds, taste preference, and dental caries in 15-years-olds. J Dent Res 1983;62:1069-72.

11. Peterson F, Rainey L. The beginning of mind in the newborn. Bull Lying-In Hosp City NY 1910;7:99-122.

12. Pfaffmann C. The pleasure of sensation. Psychol Rev 1960;67:253-68.

13. Sheiham A. Changing trends in dental caries. Int J Epidemiol 1984;13:142-7.

14. Tomita NE, Bijella VT, Lopes ES, Franco LJ. Prevalência de cárie dentária em crianças da faixa etária de 0 a 6 anos em creches de Bauru e São Paulo. Importância de fatores socioeconômicos. Rev Saúde Pública 1996;30:413-20.

15. Wyne AH, Adenubi JO, Shalan T, Khan N. Alimentary and socioeconomic characteristics of children that developed caries in a Saudian population. Pediatr Dent 1995;17-27. 